\title{
Raman spectroscopy monitoring of the cellular activities of a tissue-engineered ex vivo produced oral mucosal equivalent
}

\author{
Wen-Liang Lo, ${ }^{\text {a,b }}$ Jian-Yun Lai, ${ }^{c}$ Stephen E. Feinberg, ${ }^{d}$ Kenji Izumi, ${ }^{e}$ \\ Shou-Yen Kao, ${ }^{a, b}$ Che-Shoa Chang, ${ }^{a, b}$ Alan Linf and Huihua Kenny Chiang ${ }^{c *}$
}

\begin{abstract}
To ensure quality control and assurance in tissue engineering, noninvasive, real-time and aseptic evaluation of cell-based devices is required before product release. In this study, Raman spectroscopy was applied to monitor the cellular activities of an oral mucosa equivalent (EVPOME) produced ex vivo from cultured autogenous oral keratinocytes and acellular dermis - AlloDerm. Raman spectra showed a positive correlation of the peak area ratio of amide I $\left(1655 \mathrm{~cm}^{-1}\right) / \mathrm{phenylalanine}\left(1004 \mathrm{~cm}{ }^{-1}\right)$ with a negative linear regression $\left(R^{2}>0.95\right)$ according to the number of cultured days, especially on the 14thand $21 \mathrm{st}$ day. This work demonstrates the successful application of Raman spectroscopy for quantitatively monitoring and evaluating the maturity of EVPOME. Copyright (c) 2010 John Wiley \& Sons, Ltd.
\end{abstract}

Keywords: Raman spectroscopy; tissue engineering; ex vivo produced oral mucosal equivalent

\section{Introduction}

In Taiwan, oral cancer has the sixth highest mortality rate among all cancers. The 5-year survival rate for oral cancer patients in Taiwan is about $50 \% .^{[1]}$ The treatment regimen relies mainly on surgery, with chemotherapy and radiotherapy, usually used as adjunct therapies. Currently, the repair of small to moderate soft tissue defects after surgery uses either split-thickness skin grafts (STSGs) or full-thickness skin grafts (FTSGs) from the patient. STSG is used for covering the raw surface of the wound. The most common morbidity is scar contracture and/or infection over the donor site. Ex vivo produced oral mucosa equivalent (EVPOME) is 'surgeon friendly' in its handling characteristics and has been successfully developed as a bi-layered oral mucosa substitute by growing human oral keratinocytes on an acellular cadaveric human dermal matrix, AlloDerm (LifeCell Corporation, Branchburg, NJ). EVPOME has just completed a Federal Drug Administration (FDA) approved human clinical trial as a proof of concept for its use in periodontal treatment. ${ }^{[2-4]}$ However, according to the regulatory guidelines of the FDA, ${ }^{[5]}$ the fabricated EVPOME should be monitored before being transplanted back into the patient. Only a few techniques are capable of noninvasively determining the viability, metabolic activity and morphology of the cellular components of tissue-engineered cell-based devices during the manufacturing process. The techniques need to be administered with transparent or semitransparent scaffolds such as gels, ${ }^{[6]}$ with the introduction of gene-engineered exogenous fluorophores ${ }^{[7]}$ or with complicated isotope nuclear magnetic resonance spectroscopy. ${ }^{[8,9]}$ However, these techniques do not monitor the growth pattern of the EVPOME matrix in real time under FDA regulations. Gel scaffolds cannot be sterilized to prevent occult infections, and gene-engineered fluorophores and isotopes are prohibited from being used in cell-based products applied to humans.
An increasing number of clinical studies have demonstrated the noninvasive capability of Raman spectroscopy as a diagnostic tool in clinical applications. ${ }^{[10-12]}$ Here we use Raman spectroscopy to measure the characteristics of EVPOME during growth and fulfill the needs of noninvasive, real-time monitoring of the biomaterial during fabrication.

\section{Experimental}

\section{Procurement of human oral mucosa}

Keratinized oral mucosa was obtained from patients undergoing tooth extraction and/or minor dento-alveolar surgery. The protocol for harvesting human oral mucosal tissue was approved by the Taipei Veterans General Hospital Internal Review Board. All individuals signed informed consent forms before the tissue samples were procured.

\footnotetext{
Correspondence to: Huihua Kenny Chiang, Institute of Biomedical Engineering National Yang-Ming University, Taipei 11221, Taiwan.

E-mail: hkchiang@ym.edu.tw

a Department of Stomatology, Taipei Veterans General Hospital, Taipei, Taiwan

b Department of Dentistry, National Yang-Ming University, Taipei, Taiwan

c Institute of Biomedical Engineering, National Yang-Ming University, Taipei, Taiwan

d Department of Oral and Maxillofacial Surgery, University of Michigan Medical Center, Ann Arbor, MI 48109-0018, USA

e Division of Oral Anatomy, Niigata University Graduate School of Medical and Dental Sciences, Niigata, Japan

$\mathrm{f}$ Institute of Genetic Sciences, School of Life Sciences, National Yang-Ming University, Taipei, Taiwan
} 


\section{Cell cultures}

Primary human oral mucosal keratinocytes were obtained from keratinized oral mucosa (alveolar ridge, tuberosity, etc.) and cultured as described elsewhere. ${ }^{[4]}$

Cells were cultured in a chemically defined system, devoid of animal-derived products (EpiLife supplemented with growth factors (Cascade Biologics, Portland, OR, USA)), with a calcium concentration of $0.06 \mathrm{~mm}$ and supplemented with Fungizone $(0.375 \mu \mathrm{g} / \mathrm{ml}$; GIBCO, Grand Island, NY) and gentamicin ( $0.025 \mathrm{mg} / \mathrm{ml}$; GIBCO). Cells were maintained at $37{ }^{\circ} \mathrm{C}$ in a $5 \% \mathrm{CO} 2$ incubator. At a density of $80-90 \%$ confluence, cells were treated with $0.025 \%$ trypsin/ethylenediaminetetraacetic acid (EDTA) (Cascade Biologics) and then neutralized with $0.0125 \%$ Defined Trypsin Inhibitor (Cascade Biologics). These detached oral keratinocytes were cryopreserved in liquid nitrogen for the cell bank or were passaged for EVPOME fabrications. To get the reference Raman spectra of the cultured oral keratinocytes, we cultivated the oral keratinocytes on a sterilized silicon chip. The keratinocytes were counted and seeded at the same time and kept under the same growth conditions.

\section{EVPOME fabrications}

When the epithelial cells grown in culture flasks reached $80-90 \%$ confluence, we prepared the EVPOME.

Day 0: Cut the AlloDerm (LifeCell Corporation) to the size of each well of the 48-well cell culture plate (Corning, Lowell, MA, USA) under sterile conditions. Place the AlloDerm pieces into the $60 \times 15 \mathrm{~mm}$ polystyrene tissue culture dish and rehydrate three times with D-PBS (without $\mathrm{Ca}^{2+}$ and $\mathrm{Mg}^{2+}$ ). Pretreat the hydrated AlloDerm with human type IV collagen and store overnight at $4{ }^{\circ} \mathrm{C}$.

Day 1: Trypsinize and neutralize the cell suspension with the procedure mentioned above. Centrifuge cell suspension for $5 \mathrm{~min}$ at $24^{\circ} \mathrm{C}$ and $1000 \mathrm{rpm}$. Re-suspend cell pellet in $1.2 \mathrm{mM} \mathrm{Ca}^{2+}$ EpiLife growth medium. Seed 150000 keratinocytes per well.

Day 1-4: Change medium daily with the EVPOME submerged.

Day 4: Transfer the EVPOME to the air/liquid interface using the 6-well/Transwell plate with inserts (Organogenesis, Canton, MA).

Day 5-21: Feed every other day with $1.2 \mathrm{mM} \mathrm{Ca}^{2+}$ growth medium. On day 1, 7, 14 and 21, EVPOMEs are tested with immunohistochemistry examinations.

\section{Raman spectroscopic measurements}

Raman spectra were measured from human normal oral mucosa and the corresponding EVPOME with a confocal microscopic Raman spectroscopy system (LabRAM HR800, Jobin Yvon, Cedex, France). The system consists of an 80-cm focal length spectrometer (Jobin Yvon), a confocal microscope (BX-41, Olympus, Tokyo, Japan), a 632.8-nm, 18-mW He-Ne laser, a $1800 \mathrm{~g} / \mathrm{mm}$ grating and a liquid-nitrogen-cooled CCD detector with $1024 \times 256$ pixels.

\section{Data analysis}

Each Raman spectrum was obtained from the average of five Raman measurements with 120-s integration time from the sample to achieve a good signal-to noise-ratio. The five measurement locations were randomly selected from each natural human oral mucosa biopsy on day 1, 7, 14 and 21 during the EVPOME growth. Before the band area calculations, the original Raman spectrum was subtracted by the linear baseline of each band envelope. The procedures used to obtain the peak ratios were similar to those used by Tarnowski et al. ${ }^{[13]}$ in their analysis of the mineralization of the developing mouse calvarias by Raman spectroscopy.

\section{Results and Discussion}

\section{Raman spectroscopy of the natural human oral mucosa biopsy}

The Raman spectrum of the normal human keratinized oral mucosa is shown in Fig. 1, with weak the amide I at $1650 \mathrm{~cm}^{-1}$, the strong $\mathrm{CH}_{2}$ deformation at $1440 \mathrm{~cm}^{-1}$, broad peaks in the amide III $1200-1350 \mathrm{~cm}^{-1}$, broad $\mathrm{C}-\mathrm{OH}$ peak at $1080 \mathrm{~cm}^{-1}$ and the sharp phenylalanine peak at $1004 \mathrm{~cm}^{-1}$. All these peaks indicate contributions from proteins and lipids. To measure the Raman spectra of different layers of the epithelium, we sliced the normal oral mucosa tissues into three groups, with sections between $0-50,50-100$ and $100-150 \mu \mathrm{m}$. We found that the Raman peak intensities of amide I, $\mathrm{C}-\mathrm{H}$, amide III and $\mathrm{C}-\mathrm{OH}$ decreased from $100-150$ to $0-50 \mu \mathrm{m}$ slices as the oral keratinocytes matured. This phenomenon demonstrates the relationship between the maturities of the keratinized oral mucosa and the different components between the connective tissue and epithelial cells. The Raman band at $1004 \mathrm{~cm}^{-1}$, which remained with equal intensity in different depths of the tissue sections, represents the

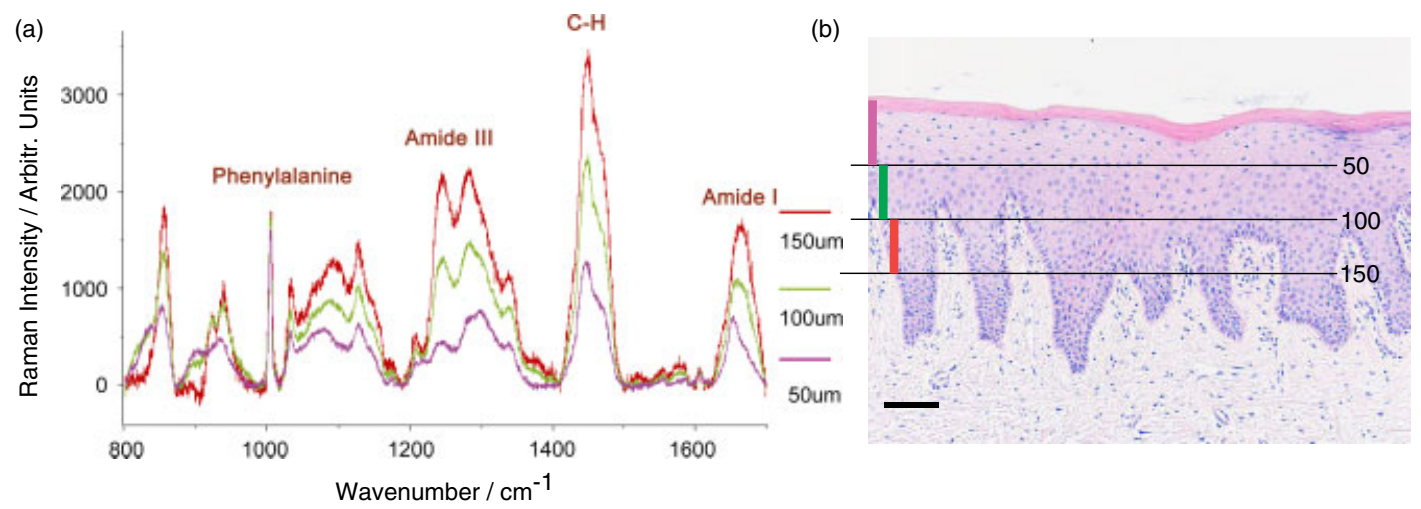

Figure 1. (a) Raman spectra of the sliced human oral keratinized oral mucosa. The four major peaks were as follows: amide I at $1650 \mathrm{~cm}^{-1}$; $\mathrm{CH}$ at $1440 \mathrm{~cm}^{-1}$; broad amide III with two peaks at 1270 and $1300 \mathrm{~cm}^{-1}$; and phenylalanine at $1001 \mathrm{~cm}^{-1}$. The amide I, C-H, amide III and $\mathrm{C}-\mathrm{OH}$ peaks decreased from the 100 - to $150-\mu \mathrm{m}$ slice to the 0 - to $50-\mu \mathrm{m}$ slice as the keratinocytes matured. (b) A representative hematoxylin and eosin-stained cross-section diagram of normal oral mucosa. Bar width: $50 \mu \mathrm{m}$. 
side chain bond (predominantly phenylalanine) of proteins. This result is in agreement with previous Raman studies of connective tissue and epithelial layer of oral mucosa. ${ }^{[14]}$ The relative intensity of the $1254 \mathrm{~cm}^{-1}$ peak in the amide III region increased when the measuring depth went down, and a right shift of the wavelength in the amide I region was noted from Fig. 1 as the measurement went deeper. These results are also the same as found in the reference cited above. ${ }^{[14]}$

\section{Raman spectroscopy of the EVPOME on day 1, 7, 14 and 21}

The Raman spectra of EVPOME (Fig. 2) showed a sharp, strong amide I band at $1655 \mathrm{~cm}^{-1}$, a strong $\mathrm{CH}_{2}$ deformation band at $1440 \mathrm{~cm}^{-1}$, broad peaks in the amide III $1200-1350 \mathrm{~cm}^{-1}$ region, a broad $\mathrm{C}-\mathrm{OH}$ peak at $1080 \mathrm{~cm}^{-1}$ and the sharp phenylalanine peak at $1001 \mathrm{~cm}^{-1}$, all of which resembled the Raman peaks from normal oral mucosa shown in Fig. 1. The relative intensity of the $1254 \mathrm{~cm}^{-1}$ peak in the amide III region decreased when the epithelial layer thickness increased. This can be explained by the fact that the signal from the connective tissue became smaller because of the masking effect of increased keratinocyte layer. ${ }^{[14]}$ We also observed the largest Raman peak intensity decrease as the EVPOME matured, except for the phenylalanine Raman peak within the 21-day course. According to our findings, the intensity of the anticipated peaks could be used as an indicator for monitoring the cellular activities of the EVPOMEs. The leftshift phenomenon of Raman spectrum in the amide I region as the EVPOMEs got more matured also was noted. In order to study the shifting of the Raman spectra, we placed emphasis on the wavenumbers between 1600 and $1700 \mathrm{~cm}^{-1}$. In Fig. 3(a), the amide I peaks were at 1652,1659 and $1666 \mathrm{~cm}^{-1}$ at the measuring depth of 50,100 and $150 \mu \mathrm{m}$ of the native oral mucosal tissue, respectively. And the amide I peaks were at 1666, 1666, 1657 and $1651 \mathrm{~cm}^{-1}$ on day 1, 7, 14 and 21 (Fig. 3(b)). According to the previous transmission electron microscopic study of the junction between basal cell layer of epidermis and the connective tissue, the anchoring fibrils were unique and abundant in this area, and the type VII collagen was analyzed to be the major component. ${ }^{[15]}$ Therefore, we measured the Raman spectra of the pure collagen VII from rat tail (Sigma-Aldrich, St. Louis, MO). It showed a peak at $1669 \mathrm{~cm}^{-1}$ in the amide I region (Fig. 3(c)), which can explain the similar peaks revealed from the Raman scanning of normal oral mucosa at $150 \mu \mathrm{m}$ depth from the junction of epithelial cells and the connective tissues and that of day 1 and 7 which had very thin layers of keratinocytes covering the cadaveric connective tissue of AlloDerm. To our best knowledge, this is the first presentation of the Raman spectra of the pure collagen VII.

\section{Univariate data analysis}

Because of the characteristics of the phenylalanine Raman spectra, further investigations were carried out using band area calculations to reveal the maturation/matrix ratios of amide I/phenylalanine, $1660 / 1004 \mathrm{~cm}^{-1}$ regions, as shown in Fig. 4. The

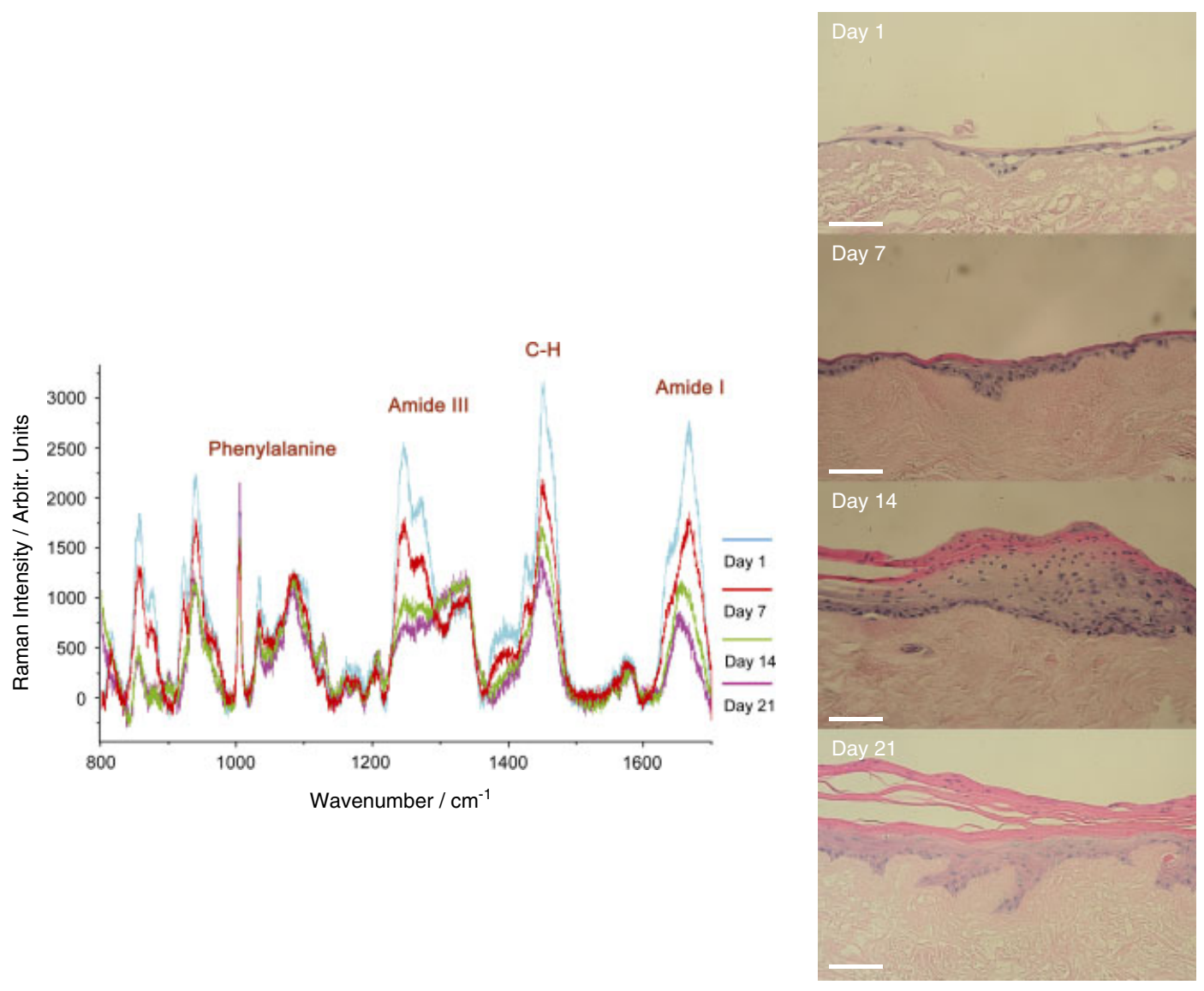

Figure 2. Raman spectra of the EVPOME. The major peaks were similar to those of the normal oral mucosa shown in Fig. 1. All the signal intensities decreased as the cultivation date increased, except phenylalanine. The adjacent representative diagrams show the hematoxylin and eosin-stained cross-section diagram of the EVPOME on different cultivation dates. Bar width: $50 \mu \mathrm{m}$. 

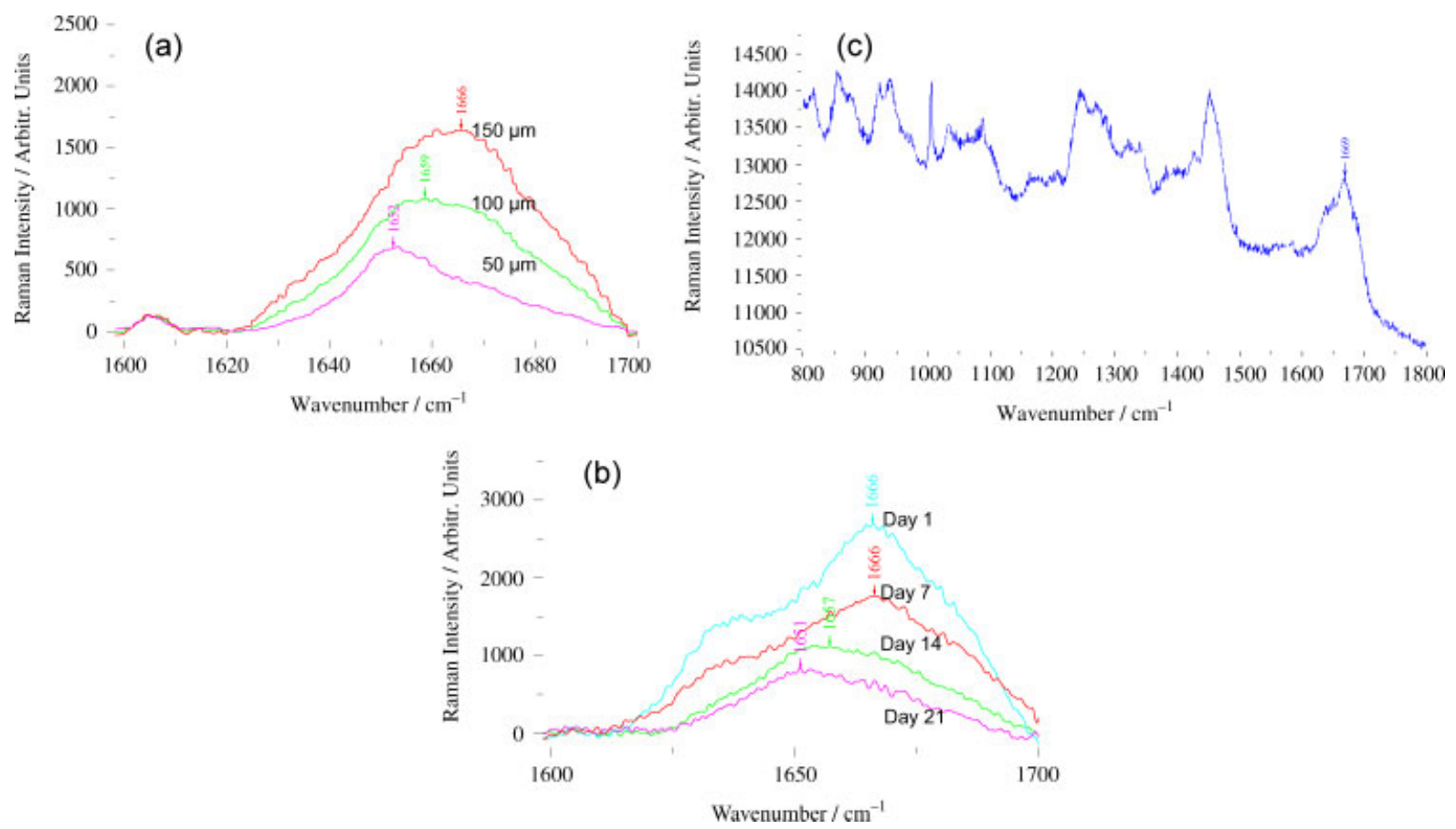

Figure 3. (a) Detailed amide I region plots in the Raman spectra of the sliced human oral keratinized oral mucosa between 1600 and $1700 \mathrm{~cm}^{-1}$. Right shift of the peak was noted when the measurement went deeper. (b) Detailed amide I region plots in Raman spectra of the EVPOME between 1600 and $1700 \mathrm{~cm}^{-1}$. Left shift of the peak was noted when the cultivation day increased. (c) Raman spectra of the pure type VII collagen from rat tail. Its amide I peak is at $1669 \mathrm{~cm}^{-1}$.
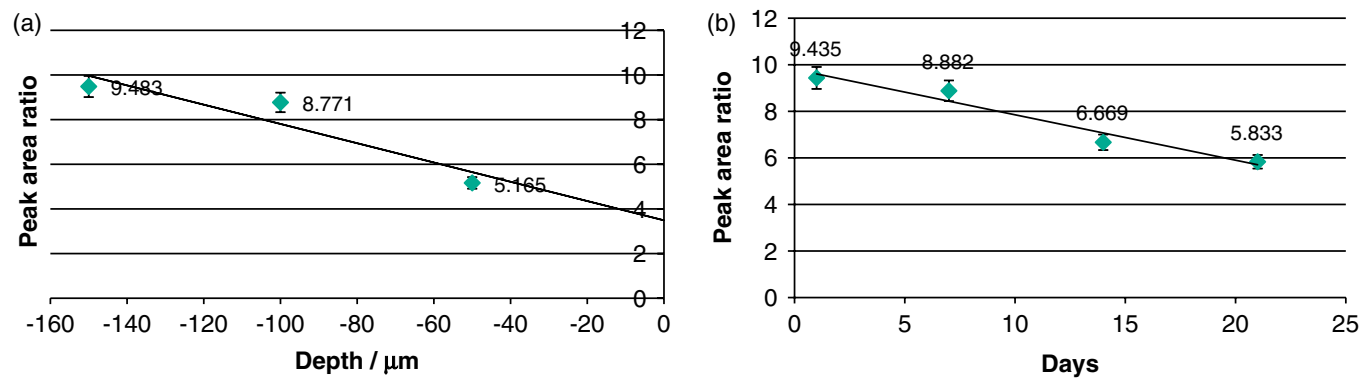

Figure 4. (a) Linear regression of the amide I/phenylalanine peak ratios at different depths of normal oral mucosa. $Y=-0.0432 x+3.4883 ; R^{2}=0.8698$. (b) Linear regression of the amide I/phenylalanine peak ratio at different cultivation dates for the EVPOMEs. $Y=-0.195 x+9.8015 ; R^{2}=0.9547$.

amide I/phenylalanine peak area ratio of normal oral mucosa at different slices had a linear regression $\left(R^{2}=0.8698\right)$. This phenomenon represented the relationship between the maturity of the cultured oral keratinocytes and the decrease in amide $\mathrm{I} /$ phenylalanine peak area ratio. A convincing linear regression $\left(R^{2}=0.9547\right)$ of the amide $\mathrm{I} /$ phenylalanine peak ratio of the EVPOMEs at different cultivating days with different maturation stages is shown in Fig. 4(b). We did not use the statistical data relating to the amide III and the $\mathrm{CH}_{2}$ group at 1245-1270 and $1445 \mathrm{~cm}^{-1}$, respectively, because peak interference was encountered in the broad amide III bands and the complex characteristics of the $\mathrm{CH}_{2}$ functional group.

The amide I band at $1645-1660 \mathrm{~cm}^{-1}$ represents an $\alpha$ helix structure in proteins, whereas the amide I band between $1665-1680$ and $1660-1670 \mathrm{~cm}^{-1}$ represents the $\beta$-sheet structure and random coil. ${ }^{[16,17]}$ In epithelial tissue, the most abundant proteins that possess an $\alpha$-helix structure are collagens, ${ }^{[18]}$ and the Raman spectrum is very sensitive to secondary structure of the polypeptide chain in the amide I region. ${ }^{[19]}$ The type VII collagen fibril, especially, is composed of three identical alpha collagen chains and is restricted to the basement zone beneath stratified squamous epithelia. It functions as an anchoring fibril between the external epithelia and the underlying stroma. ${ }^{[20]}$ Our data showed the $1669 \mathrm{~cm}^{-1}$ peak in the amide I region of pure collagen VII, and this can explain the $1666 \mathrm{~cm}^{-1}$ peaks in the amide I region revealed in the depth of normal oral mucosa at $150 \mu \mathrm{m}$ from the junction of epithelial layer and the underlying stroma and that of day 1 and 7 EVPOMEs. What about the left shift of peaks in the amide I region detected in the depth of normal oral mucosa of 50 and $100 \mu \mathrm{m}$ and that of day 14 and 21 EVPOMEs? De Veld et al. and Malini et al. obtained similar results, but no definite explanation was given. ${ }^{[14,21]}$ Our collaborating team found recently that the vascular endothelial growth factor (VEGF), which is produced by keratinocytes and has been known to be constitutively secreted from keratinocytes in monolayer cultures, ${ }^{[22-24]}$ increased in association with increasing numbers of viable cells in the EVPOME and could be a screening marker of the metabolic activity. ${ }^{[25]}$ The secondary structure of VEGF is 
smaller than that of collagen and has more $\alpha$-helical segments than the collagen (coiled coil). We have arrived at a preliminary conclusion regarding the peak shift noted in both normal oral mucosa and three-dimensional EVPOME construct.

Phenylalanine is an essential amino acid that is found naturally in the milk of mammals. Phenylalanine's conversion into tyrosine is catalyzed by the enzyme phenylalanine hydroxylase $(\mathrm{PAH}$; phenylalanine 4-monooxygenase, EC 1.14.16.1), which is mainly expressed in the liver. PAH activity has also been detected in the skin. ${ }^{[26]}$ In keratinocytes, tyrosine is the substrate for tyrosine hydroxylase, leading to the production of catecholamines. ${ }^{[27]}$ The stable peak intensity of the phenylalanine band at $1004 \mathrm{~cm}^{-1}$ could be explained by the active catalysis of phenylalanine by viable keratinocytes grown on the EVPOME. Meanwhile, the proteins/peptides have phenylalanine side chain, also giving rise to the Raman peak at $1001 \mathrm{~cm}^{-1}$, and their roles in the cellular activities are different ${ }^{[28,29]}$ and therefore we could not arrive at a conclusion in our study.

\section{Conclusions}

We have characterized a linear regression relationship during the EVPOME maturation process by monitoring the Raman signals of amide land phenylalanine. A close correlation $\left(R^{2}=0.95\right)$ between the amide I/phenylalanine ratio and the EVPOME culturing day was established using simple linear regression statistics. The use of Raman vibrational spectroscopy is very important to the understanding and monitoring of oral keratinocyte maturation during the EVPOME fabrication process. Raman spectroscopy provides good quantitative evidence for the maturation of the three-dimensional construct and is therefore suitable for noninvasive, real-time monitoring in soft tissue engineering.

\section{Acknowledgements}

This study was supported by research grants from the National Science Council (NSC-96-3111-B-075-001-MY3), Taipei Veterans General Hospital (VGH-97-01-24A, 98F-004) and the Yen Tjing Ling Medical Foundation (Cl-97-18, Cl-98-18).

\section{References}

[1] W. L. Lo, S. Y. Kao, L. Y. Chi, Y. K. Wong, R. C. Chang, J. Oral Maxillofac. Surg. 2003, 61, 751

[2] K. Izumi, S. E. Feinberg, A. lida, M. Yoshizawa, Int. J. Oral Maxillofac. Surg. 2003, 32, 188 .
[3] K. Izumi, J. Song, S. E. Feinberg, Cells Tissues Organs 2004, 176, 134

[4] K. Izumi, H. Terashi, C. L. Marcelo, S. E. Feinberg, J. Dent. Res. 2000, $79,798$.

[5] G. MacKay, Regen. Med. 2006, 1, 169.

[6] W. Sun, R. Xu, W. Hu, J. Jin, H. A. Crellin, J. Bielawski, Z. M. Szulc, B. H. Thiers, L. M. Obeid, C. Mao, J. Invest. Dermatol. 2008, 128, 389.

[7] J.S. Blum, J.S. Temenoff, H. Park, J. A. Jansen, A. G. Mikos, M. A. Barry, Biomaterials 2004, 25, 5809.

[8] C. L. Stabler, R. C. Long, A. Sambanis, I. Constantinidis, Tissue Eng. 2005, 11, 404

[9] K. W. Ng, D. T. Leong, D. W. Hutmacher, Tissue Eng. 2005, 11, 182.

[10] S. Sigurdsson, P. A. Philipsen, L. K. Hansen, J. Larsen, M. Gniadecka, H. C. Wulf, IEEE Trans. Biomed. Eng. 2004, 51, 1784.

[11] M. Gniadecka, P. A. Philipsen, S. Sigurdsson, S. Wessel, O. F. Nielsen, D. H. Christensen, J. Hercogova, K. Rossen, H. K. Thomsen, R. Gniadecki, L. K. Hansen, H. C. Wulf, J. Invest. Dermatol. 2004, 122, 443.

[12] Z. Huang, A. McWilliams, H. Lui, D. I. McLean, S. Lam, H. Zeng, Int. J. Cancer. 2003, 107, 1047.

[13] C. P. Tarnowski, M. A. Ignelzi Jr, M. D. Morris, J.BoneMiner. Res. 2002 $17,1118$.

[14] D. C. de Veld, T. C. Bakker Schut, M. Skurichina, M. J. Witjes, J. E. Van der Wal, J. L. Roodenburg, H. J. Sterenborg, Lasers Med. Sci. 2005 19,203

[15] T. Osawa, M. Abe, A. Morigami, Y. Nozaka, Arch. Oral Biol. 2000, 45, 419.

[16] K. Guze, M. Short, S. Sonis, N. Karimbux, J. Chan, H. Zeng, J. Biomed. Opt. 2009, 14, 014016.

[17] P. R. Carey, Biochemical Applications of Raman and Resonance Raman Spectroscopies, Academic Press: New York, 1982.

[18] A. Nanci, Ten Cate's Oral Histology (7th edn), Mosby: St. Louis, 2007.

[19] B. G. Frushour, J. L. Koenig, Biopolymers 1975, 14, 379.

[20] R. E. Burgeson, J. Invest. Dermatol. 1993, 101, 252.

[21] R. Malini, K. Venkatakrishna, J. Kurien, K. M. Pai, L. Rao, V. B. Kartha, C. M. Krishna, Biopolymers 2006, 81, 179.

[22] A. M. Szpaderska, C. G. Walsh, M. J. Steinberg, L. A. DiPietro, J. Dent. Res. 2005, 84, 309.

[23] S. Boussat, S. Eddahibi, A. Coste, V. Fataccioli, M. Gouge, B. Housset, S. Adnot, B. Maitre, Am. J. Physiol. Lung Cell. Mol. Physiol. 2000, 279, L371

[24] A. Coste, L. Brugel, B. Maitre, S. Boussat, J. F. Papon, L. Wingerstmann, R. Peynegre, E. Escudier, Eur. Respir. J. 2000, 15, 367.

[25] Q. Xu, K. Izumi, T. Tobita, Y. Nakanishi, S. E. Feinberg, J. Oral Maxillofac. Surg. 2009, 67, 1256

[26] K. U. Schallreuter, J.M. Wood, M. R. Pittelkow, M. Gutlich, K. R. Lemke, W. Rodl, N. N. Swanson, K. Hitzemann, I. Ziegler, Science 1994, 263, 1444.

[27] K. U. Schallreuter, J. M. Wood, I. Ziegler, K. R. Lemke, M. R. Pittelkow, N. J. Lindsey, M. Gutlich, Biochim. Biophys. Acta 1994, 1226, 181.

[28] A.Comer, M. Hoffmann, L.-A. Hoffmann, AA61K3512Fl; Casimir Jones SC, Strata Tech Corporation, 2009.

[29] C. D. Kane, N. R. Coe, B. Vanlandingham, P. Krieg, D. A. Bernlohr, Biochemistry 1996, 35, 2894. 